\title{
Nursing factors in performing daily interruption of sedation in a large intensive care unit
}

\author{
L Roberts ${ }^{*}$, C Snelson \\ From ESICM LIVES 2015 \\ Berlin, Germany. 3-7 October 2015
}

\begin{abstract}
Introduction
Sedation is a fundamental aspect of management of Intensive Care Unit (ICU) patients. Improvements in sedation practice (including daily interruption of sedation [DIS]) have been strongly associated with decreased ICU length of stay and decreased duration of mechanical ventilation [1]. A previous intra-departmental audit had shown scope for improving DIS performance [2]. Nursing care factors impact on efficacious performance of DIS. However, there is limited qualitative evidence determining the specific factors to target in order to optimise patient care.
\end{abstract}

\section{Objectives}

Identification of modifiable factors within the nursing care provided to ICU patients to allow optimisation of the performance of DIS.

\section{Methods}

This qualitative study was performed in a large 75 bedded ICU with over 450 nursing staff. At the time of study, there was no specific protocol for sedation management within the ICU. Questionnaires were anonymously distributed and collected from all nursing staff members on duty on 6 shifts (day and night). Free text answers underwent qualitative content analysis, coding key themes and concepts. Three specific categories of factors were examined: knowledge, training and clinical practice.

\section{Results}

$30 \%$ of participants $(60 ; n=200)$ responded to the questionnaire. There was a heterogeneous awareness of the benefits of DIS amongst respondents. This was exemplified by $56.7 \%$ stating that DIS was an opportunity to assess neurology and $15 \%$ noting it as an opportunity to assess the potential for extubation. The most commonly stated disadvantages of DIS were: risk to patient (46.7\%), agitation $(28.3 \%)$ and causing the patient distress (16.7\%) - demonstrating similar heterogeneity. The majority $(83 \%)$ of respondents were unaware of any research concerning DIS. 55.9\% were untrained in DIS with $79.3 \%$ desiring training/further training. $48.3 \%$ stated that doctors led DIS whilst $45 \%$ stated that this role was a joint doctor-nurse responsibility. $68.3 \%$ felt that it should be led jointly. $61.7 \%$ were unaware of the recommended sedation score target during a DIS.

\section{Conclusions}

A number of nursing factors were identified that may be impacting on performance of DSI and patient outcomes. These could largely be rectified with systematic training and education, with the nursing cohort being receptive to this. Introducing a protocol for management of sedation may also help mitigate the human factors impacting on the performance of DSI.

Published: 1 October 2015

\section{References}

1. Jackson DL, Proudfoot CW, Cann KF, Walsh T: A systematic review of the impact of sedation practice in the ICU on resource use, costs and patient safety. Crit Care 2010, 14(2):R59.

2. Roberts L, Snelson C: An Audit of the Performance of Sedation Holds in QEHB ICU. 2014, [Unpublished clinical audit]. Queen Elizabeth Hospital Birmingham (University Hospitals Birmingham NHS Foundation Trust)..

doi:10.1186/2197-425X-3-S1-A323

Cite this article as: Roberts and Snelson: Nursing factors in performing daily interruption of sedation in a large intensive care unit. Intensive Care Medicine Experimental 2015 3(Suppl 1):A323. 